\title{
BMJ Open Quality DC cardioversion of atrial fibrillation and atrial flutter in the emergency department: improving specialist protocols for the generalist
}

\author{
Alexander Carpenter, Sabrina Sargent
}

To cite: Carpenter A, Sargent S. DC cardioversion of atrial fibrillation and atrial flutter in the emergency department: improving specialist protocols for the generalist. BMJ Open Quality 2018;4:e000260. doi:10.1136/ bmjoq-2017-000260

Received 10 May 2018 Revised 26 October 2018 Accepted 8 November 2018

Check for updates

(C) Author(s) (or their employer(s)) 2018. Re-use permitted under CC BY-NC. No commercial re-use. See rights and permissions. Published by BMJ.

Department of Cardiology, Severn Deanery, Bristol, UK

Correspondence to Dr Alexander Carpenter; alexander.carpenter@doctors. org.uk

\section{ABSTRACT}

Background Direct current cardioversion (DCCV) is a safe and effective treatment for recent-onset atrial fibrillation (AF) or flutter and when performed in the emergency department (ED), it can provide an excellent treatment option for patients as well as reducing unnecessary hospital admissions and healthcare costs. However, appropriate periprocedural anticoagulation is absolutely essential to reduce the risk of adverse outcomes, chiefly thromboembolic stroke. Our intention was for $100 \%$ of patients undergoing DCCV in the ED to receive appropriate periprocedural anticoagulation.

Method We aimed to assess local practice with regards to periprocedural anticoagulation with a 1-year retrospective audit. We then undertook to deliver a multimodality educational programme in addition to producing new local protocols. Stakeholders were engaged within the cardiology, emergency medicine and governance departments as well as trust quality improvement team. This was undertaken across three PDSA cycles with prospective data collection on a rolling monthly basis with the use of real-time run charts, fed back to the ED. Teaching was delivered on a small group, electronic as well as departmental level, and a new protocol was created and delivered to guide clinicians in the management of patients with AF or flutter.

Results While initial rates of periprocedural anticoagulation were suboptimal (with only $72 \%$ of eligible patients anticoagulated), following our programme of continuous monitoring and intervention, this steadily rose over the project timeline, achieving a high of $91 \%$ at the point of last data collection.

Conclusion We should champion the high number of these procedures carried out in the ED setting, a pressured environment with multiple competing challenges. However, local protocols should reflect best-practice guidance regarding decision-making around selecting rate versus rhythm control strategies, appropriate use of medication and eligibility for anticoagulation as per individualised thrombotic risk. This will allow us to deliver effective interventions in a safe, patient-centred approach.

\section{PROBLEM IDENTIFICATION}

Direct current cardioversion (DCCV) of atrial fibrillation (AF) is a safe and effective method to restore sinus rhythm in patients presenting to the emergency department (ED). It can improve symptoms and prevent deterioration, as well as proving cost-effective.

However, anecdotal reports locally suggested that adherence to recently updated guidelines was variable. In particular, adherence to advice regarding periprocedural anticoagulation, important to reduce the risk of subsequent stroke, had been flagged up.

We sought to assess this on an ongoing basis, provide educational tools, deliver sessions and implement systems change in the form of a new protocol to ensure $100 \%$ of eligible patients received appropriate periprocedural anticoagulation.

\section{BACKGROUND}

$\mathrm{AF}$ is the most common sustained arrhythmia, with a prevalence in the general population of approximately $1 \%{ }^{1}$ It accounts for at least 3\% of National Health Service (NHS) expenditure, with a steadily increasing $10 \%$ of all UK emergency admissions related to AF or atrial flutter. ${ }^{2}$

NHS England provides guidance for AF as an 'ambulatory care sensitive condition', that is, a condition where appropriate management in the acute setting can reduce the need for hospital admission. Indeed, many patients presenting with AF or flutter with symptoms or uncontrolled ventricular rate can be safely treated in the ED with a synchronised direct current (DC) cardioversion, an electric shock therapy which, if successful, restores a normal sinus heart rhythm and allows discharge home from the ED. This treatment has been shown to be safe $^{34}$ and effective, ${ }^{5}$ and should be encouraged, where local services allow.

A key consideration at the time of DC cardioversion is the need to anticoagulate to reduce the risk of subsequent stroke. Although it has long been established that a new-onset AF or atrial flutter within 48 hours is likely safe to cardiovert, new data have raised questions about the role 


\section{Driver Diagram}

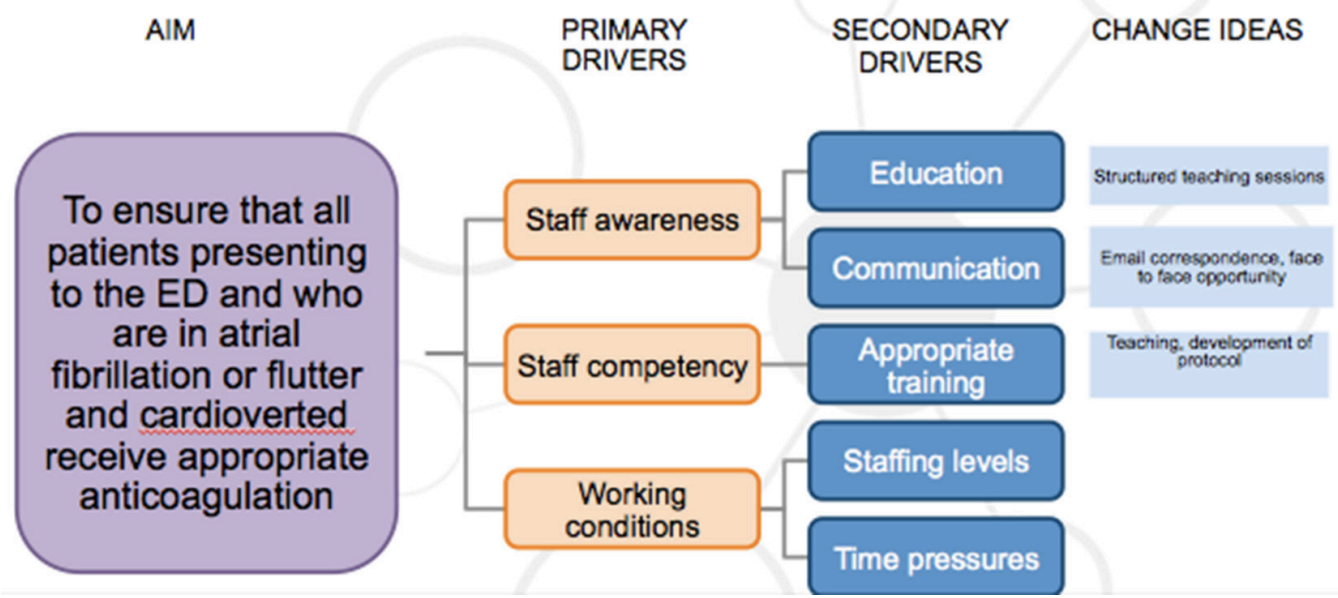

Figure 1 Driver diagram: a graphic showing the project aims, primary and secondary drivers for change as well as change ideas. ED, emergency department.

of postprocedural anticoagulation ${ }^{3}$ and this is reflected in the latest European and British guidelines-with the decision to anticoagulate based on the patient's individualised thromboembolic risk, using the 'CHADsVASc' risk score. ${ }^{6-8}$ Within our own institution, we sought to assess and improve the proportion of ED cardioversions which adhered to this updated guidance to improve compliance with best practice and maximise patient safety.

\section{BASELINE MEASUREMENT}

We undertook an initial retrospective analysis of all patients cardioverted for $\mathrm{AF}$ or flutter in the $\mathrm{ED}$ over a 1 -year period. We then prospectively recorded these data on an ongoing basis.
Our baseline retrospective measurements revealed that over a 1-year period, the number of cardioversions in ED was substantial, that is, 57 , which should be celebrated as this is likely to represent a reduction in the burden of unnecessary hospital admissions. Sixty-one per cent of these patients were men, with a mean age of 56 (range 23-88).

Of the 57 patients who underwent cardioversion, $32(56 \%)$ of these were eligible for anticoagulation, according to their calculated CHADsVASc score to assess thromboembolic risk. Twenty-three $(72 \%)$ of these patients were started on post-procedural anticoagulation, a suboptimal figure which probably represents variable staff familiarity with updated national guidance as well as a lack of clear local guidelines on the subject.

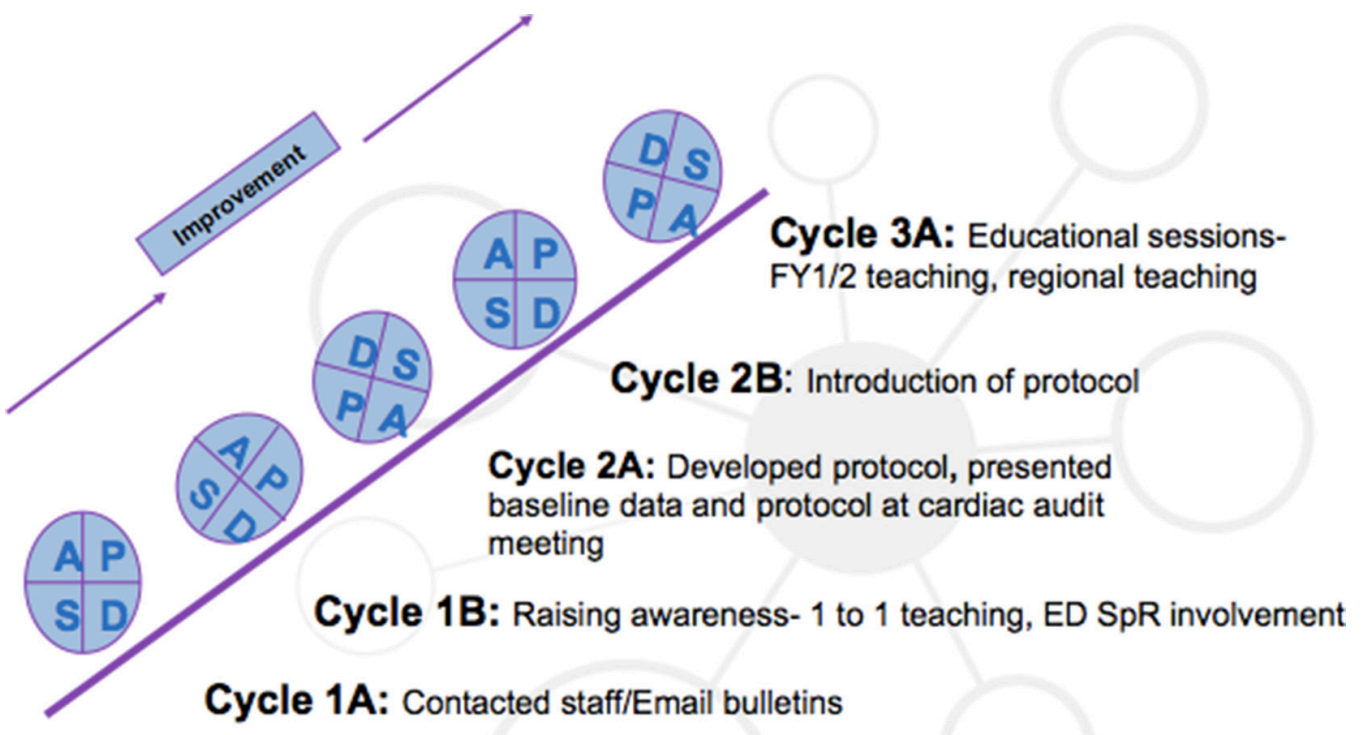

Figure 2 PDSA cycles: the five PDSA cycles illustrated with the main summary changes listed for each stage. ED, emergency department; PDSA, plan, do, study, act; SpR, specialist registrar. 


\section{DESIGN}

Our overall aim was that $100 \%$ of DC cardioversions in the $\mathrm{ED}$ for $\mathrm{AF}$ or flutter receive appropriate periprocedural anticoagulation.

We engaged with various stakeholders. The cardiology department including the local arrhythmia group, were consulted with to establish best practice and expectations for an updated guideline.

We engaged with the ED and worked with an ED trainee to understand possible factors influencing the management of these patients, as well as the potential approaches and obstacles to improving the process. We also engaged our Trust's local quality improvement team, who provided invaluable assistance in clarifying our measures and process, as well as engaging the relevant individuals from the audit and coding departments.

We produced a driver diagram (figure 1) which explores some of the factors influencing whether patients received appropriate anticoagulation and some of the change ideas surrounding this. Having consulted widely, we felt that severe time pressures within the ED, coupled with a very variable range of levels of seniority and experience, as well as the presence of temporary staff, might contribute to the variable adherence to contemporary guidelines. Similarly, we felt that a lack of clear local guidelines (which were present but out-dated and no longer in keeping with contemporary best practice) further compounded the issue, and that clear, easily accessible local guidance coupled with emailed, large group and individual teaching sessions would be the best method of disseminating current best practice.

\section{STRATEGY}

PSDA cycle 2: We attempted to reinforce our idea and interventions by designing an evidence-based and userfriendly protocol to further improve and standardise care for patients. The most current guidelines were used to create this protocol and approval from the local cardiology department was sought prior to implementation. Data were prospectively collected from the medical notes 3 months after implementation of the protocol. This demonstrated a good improvement showing among 36 patients cardioverted, in whom $22(61 \%)$ had an indication for cardioversion, and 20 (91\%) of these were managed appropriately. It was evident that senior practices were the most challenging to change. For new staff, the protocol provided an easy-to-follow guideline as part of the departmental guidelines all new ED staff were introduced to at induction.

PSDA cycle 3: after achieving a good response from the majority of staff, we attempted to sustain improvements through further promotion of our project within and outside the ED. This was achieved by educational means with the use of presentations and posters throughout the department. Ongoing data collection showed that this improvement was maintained. The updated protocol remains in place for ED as well as the rest of the hospital (figure 2 ).

\section{RESULTS}

From our electronic patient coding system, we obtained valuable information regarding patient assessments, including time and date seen, grade of care provider but more importantly their stroke risk (using the CHADsVASc score) and whether they were anticoagulated appropriately or not and followed up on discharge. Patients with a CHADsVASc score of $>1$ if man or $>2$ if woman were deemed eligible for anticoagulation providing there was no significant risk to treatment (determined using the HASBLED score or other documented contraindication or a documented clinical decision not to anticoagulate). Acceptable methods of anticoagulation included low-molecular-weight heparin, warfarin or any direct oral anticoagulant (DOAC).

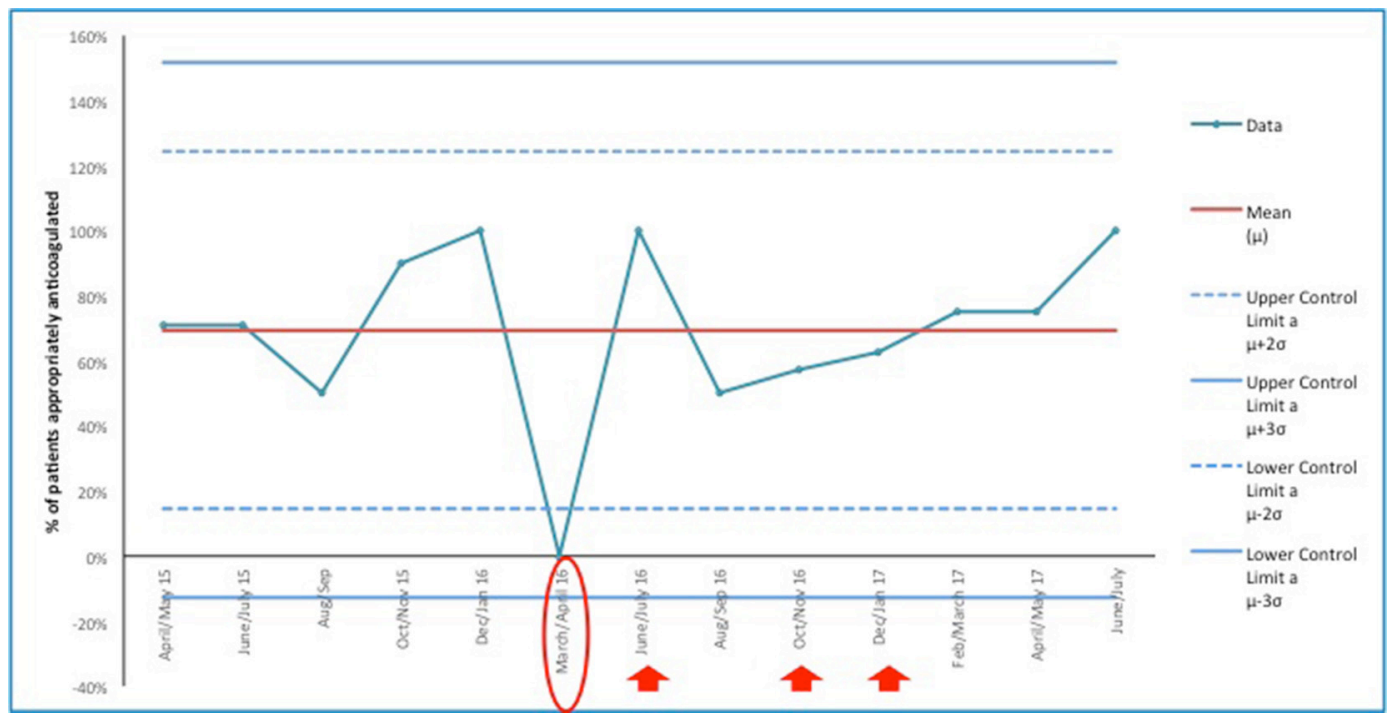

Figure 3 Run chart showing the change in our primary outcome measure (percentage of patients appropriately anticoagulated) over time, with the raw data, mean average and upper and lower control limits displayed. 
Atrial fibrillation and Atrial Flutter in the Emergency Department

Approved by Local Arrhythmia Group Aug 2016

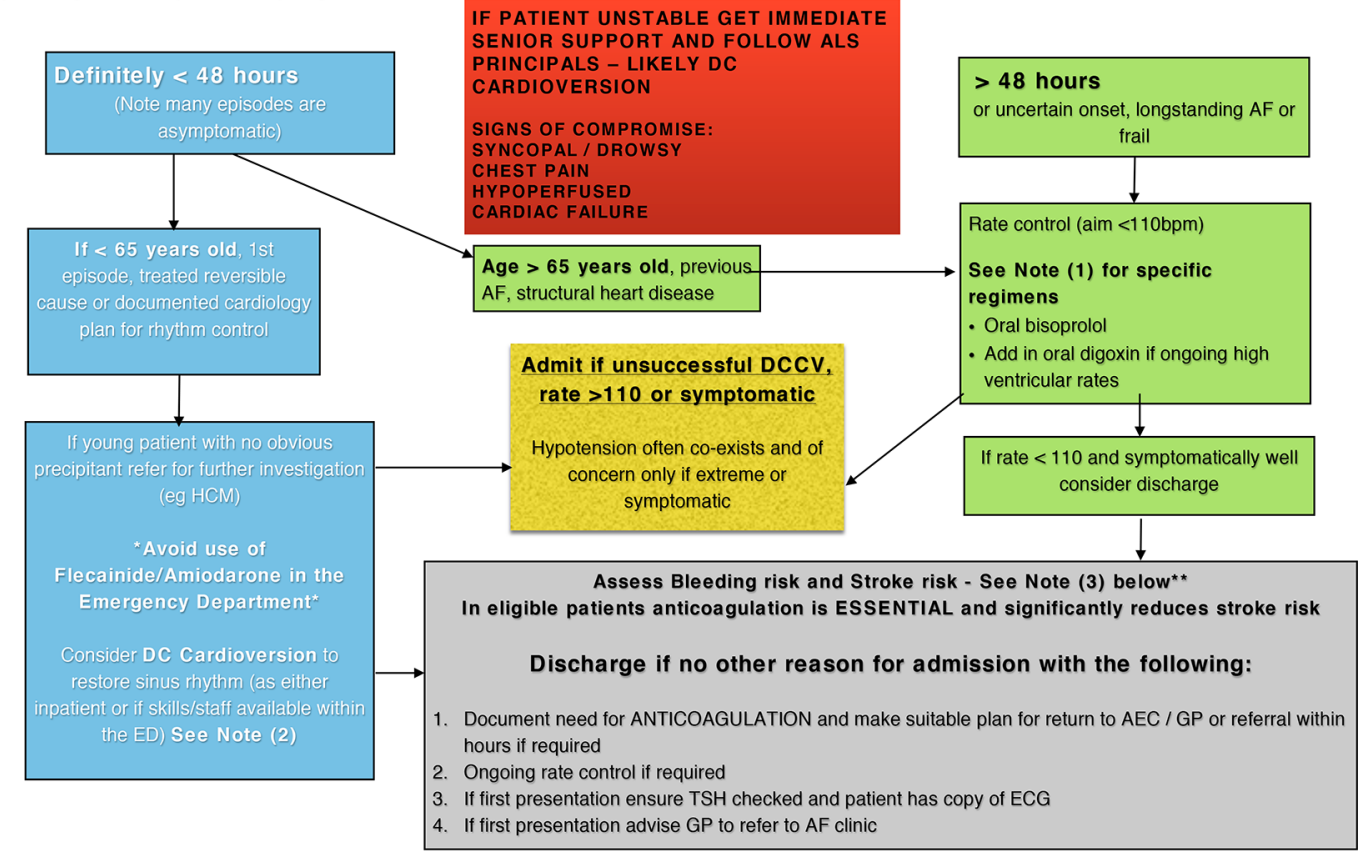

\begin{tabular}{l}
$\begin{array}{l}\text { Note (1) Rate Control } \\
\text { Bisoprolol - Give } 2.5 \mathrm{mg} \text { stat then } 2.5 \mathrm{mg} \mathrm{BD} \text { on discharge, only avoid if acute wheezy exacerbation } \\
\text { Digoxin - Equivalent loading if oral or IV therefore } 500 \mathrm{mcg} \text { PO stat followed by } 500 \mathrm{mcg} 12 \text { hours later. (If required then clearly needs to be as inpatient). } \\
\text { Patients may be discharged on } 125 \mathrm{mcg} \text { OD if required }\end{array}$ \\
\hline $\begin{array}{l}\text { Note (2) Rhythm Control: DCCV } \\
\text { Within the emergency department up to x } 3 \mathrm{DCCV} \text { at } 150 \mathrm{~J} \text { - ALWAYS with sedation and senior support. Appropriate pre and post procedural anticoagulation. }\end{array}$ \\
\hline $\begin{array}{l}\text { Note (3) Stroke risk: In eligible patients anticoagulation is ESSENTIAL and significantly reduces stroke risk } \\
\text { Calculate CHADSVASC and HASBLED scores. Consider commencing anticoagulation if appropriate within the ED otherwise refer to AEC. } \\
\text { NOACs (apixaban, dabigatran or rivaroxaban) are a simple and effective mode of anticoagulation in AF. Please consider a stat dose of low } \\
\text { molecular weight heparin pre-shock. }\end{array}$ \\
\end{tabular}

CHA2DS2VASc

\begin{tabular}{|c|c|}
\hline \multicolumn{1}{|c|}{ Risk factor } & Yes/No \\
\hline Congestive Heart Failure & Yes $\square$ No \\
\hline Hypertension & Yes $\square$ No \\
\hline Age $>75$ & Yes $\square$ No \\
\hline Age $65-74$ & Yes $\square$ No \\
\hline Diabetes Mellitus & Yes $\square$ No \\
\hline Previous stroke or TIA & Yes $\square$ No \\
\hline \multicolumn{2}{|c|}{ Female } \\
\hline \multicolumn{2}{|c|}{ Yes $\square$ No } \\
\hline Total Points & Action (Unless C/I) \\
\hline If 1 or more males & Anticoagulate with NOAC \\
\hline $\begin{array}{l}\text { If } 2 \text { or more in } \\
\text { females }\end{array}$ & Anticoagulate with NOAC \\
\hline
\end{tabular}

HASBLED

\begin{tabular}{|c|c|c|}
\hline Risk factor & Yes/No & Points \\
\hline Hypertension & Yes $\square$ No $\square$ & 1 \\
\hline Abnormal Liver +/- renal function & Yes $\square$ No $\square$ & 1 each \\
\hline Stroke & Yes $\square$ No $\square$ & 1 \\
\hline Bleeding tendency & Yes $\square$ No $\square$ & 1 \\
\hline Labile INR & Yes $\square$ No $\square$ & 1 \\
\hline$>65$ years old & Yes $\square$ No $\square$ & 1 \\
\hline Drug and or alcohol use & Yes $\square$ No $\square$ & 1 each \\
\hline & Total & \\
\hline
\end{tabular}

$$
\begin{gathered}
\text { Novel oral anticoagulants (NOACs): } \\
\text { Rivaroxaban } 20 \mathrm{mg} \text { OD if } \mathrm{CrCl}>50 \\
\text { Apixaban } 2.5 \mathrm{mg} \text { or } 5 \mathrm{mg} \mathrm{BD} \\
\text { dependent on age, weight and renal } \\
\text { function }
\end{gathered}
$$

\begin{tabular}{|l|}
\hline Total Points \\
\hline If $>$ or $=$ to 3 consider high risk \\
\hline $\begin{array}{l}\text { Don't rule out anticoagulation only based on } \\
\text { risk of falling }\end{array}$ \\
\hline
\end{tabular}

Figure 4 Protocol for management of AF and atrial flutter in the ED. AEC, Ambulatory Emergency Care; AF, atrial fibrillation; DC, direct current; DCCV, direct current cardioversion; ED, emergency department; GP, general practitioner; HCM, hypertrophic cardiomyopathy ; NOAC, novel oral anticoagulant; TSH, thyroid stimulating hormone.

Data were collected using the same method described above on a monthly basis from April 2016 to July 2017 to measure the effects of our interventions. Data were plotted on a real-time run chart to establish whether changes were made due to our targeted interventions or due to other variations.

For the 1-year retrospectively assessed period from April 2015 to 2016, 57 patients were cardioverted in the ED. Of theses, $32(56 \%)$ patients were eligible for anticoagulation with no obvious contraindications. However, only $23(72 \%)$ of these patients were anticoagulated or a plan made for this.

Following the introduction of our small group teaching sessions during PDSA cycle 1, we began to prospectively collect data for the subsequent year (see run chart-this timepoint marked with red circle). 
PDSA cycle 2 saw the creation, refinement and introduction of a formal Trust protocol for the procedure and its marketing locally.

Cycle 3 saw teaching delivered on various formal teaching programmes (foundation teaching, local Emergency Medicine and Primary Care organised teaching sessions).

The run chart plots the proportion of patients (as a percentage of total eligible) who were appropriately anticoagulated over the QI project timeline. As can be seen, this proportion increased steadily following our interventions (marked with arrows) and continues to do so. Indeed, at the most recent data collection point, 20 out of $22(91 \%)$ eligible patients undergoing the procedure had been appropriately anticoagulated, a significant improvement (figure 3). The updated protocol itself is available as an attachment (figure 4).

\section{LESSONS AND LIMITATIONS}

This project initially ran into challenges with regards to instituting change in a large organisation. Thankfully, departmental contacts allowed us to progress appropriately, especially in terms of updating outdated electronic clinical guidance.

One major limitation is that our project was based on ED coding in order to inform our data collection, this relies on accurate clinician coding and there may have been a small number of cases missed as a result. Hopefully, this would not affect our overall conclusions as the proportion of patients anticoagulated would arguably be unchanged.

\section{CONCLUSION}

DCCV is a safe and effective treatment for recent-onset AF or flutter and when performed in the ED, it can provide an excellent treatment option for patients as well as reducing unnecessary hospital admissions and healthcare costs. However, appropriate periprocedural anticoagulation is absolutely essential to reduce the risk of adverse outcomes, chiefly thromboembolic stroke.

We should champion the high number of these procedures carried out in the ED setting, a pressured environment with multiple competing challenges. However, local protocols should reflect best-practice guidance regarding decision-making around selecting rate versus rhythm control strategies, appropriate use of medication and eligibility for anticoagulation as per individualised thrombotic risk (the CHADsVASc score).

Acknowledgements The authors acknowledge Dr Nicol Vaidya, Associate Specialist in Cardiology, and Dr Faye Noble, Consultant in Emergency Medicine, for their contributions and invaluable support.

Contributors AC and SS: conception, design and implementation of the project; results analysis as well as final write-up.

Funding The authors have not declared a specific grant for this research from any funding agency in the public, commercial or not-for-profit sectors

Competing interests None declared.

Patient consent Not required.

Ethics approval This project was undertaken as a service evaluation in keeping with local Trust guidelines and was approved by our local quality improvement academy as an appropriate, valuable and ethical project.

Provenance and peer review Not commissioned; externally peer reviewed.

Open access This is an open access article distributed in accordance with the Creative Commons Attribution Non Commercial (CC BY-NC 4.0) license, which permits others to distribute, remix, adapt, build upon this work non-commercially, and license their derivative works on different terms, provided the original work is properly cited, appropriate credit is given, any changes made indicated, and the use is non-commercial. See: http://creativecommons.org/licenses/by-nc/4.0/.

\section{REFERENCES}

1. Go AS, Hylek EM, Phillips KA, et al. Prevalence of diagnosed atrial fibrillation in adults: national implications for rhythm management and stroke prevention: the AnTicoagulation and Risk Factors in Atrial Fibrillation (ATRIA) Study. JAMA 2001;285:2370-5.

2. NHS England. Emergency admissions for Ambulatory Care Sensitive Conditions - characteristics and trends at national level. London: NHS England, 2014.

3. Airaksinen KE, Grönberg T, Nuotio I, et al. Thromboembolic complications after cardioversion of acute atrial fibrillation: the FinCV (Finnish CardioVersion) study. J Am Coll Cardiol 2013;62:1187-92.

4. de Paola AA, Figueiredo E, Sesso R, et al. Effectiveness and costs of chemical versus electrical cardioversion of atrial fibrillation. Int $J$ Cardiol 2003;88:157-66.

5. Michael JA, Stiell IG, Agarwal S, et al. Cardioversion of paroxysmal atrial fibrillation in the emergency department. Ann Emerg Med 1999;33:379-87.

6. Kirchhof P, Benussi S, Kotecha D, et al. 2016 ESC Guidelines for the management of atrial fibrillation developed in collaboration with EACTS. Eur J Cardiothorac Surg 2016;50:e1-88.

7. NICE. Atrial fibrillation: management | Guidance and guidelines. London: NICE, 2014.

8. Lip GY, Nieuwlaat R, Pisters R, et al. Refining clinical risk stratification for predicting stroke and thromboembolism in atrial fibrillation using a novel risk factor-based approach: the euro heart survey on atrial fibrillation. Chest 2010;137:263-72. 